\title{
Pattern Recognition using Artificial Neural Network
}

\author{
Poonam Dabas \\ Department of Computer Science and Engineering \\ University Institute of Engineering and Technology \\ Kurukshetra University \\ Kurukshetra, India
}

\author{
Umesh Kumar \\ Department of Computer Science and Engineering \\ University Institute of Engineering and Technology \\ Kurukshetra University \\ Kurukshetra, India
}

\begin{abstract}
An artificial neural network (ANN) usually called neural network. It can be considered as a resemblance to a paradigm which is inspired by biological nervous system. In network the signals are transmitted by the means of connections links. The links possess an associated way which is multiplied along with the incoming signal. The output signal is obtained by applying activation to the net input $\mathrm{NN}$ are one of the most exciting and challenging research areas. As ANN mature into commercial systems, they are likely to be implemented in hardware. Their fault tolerance and reliability are therefore vital to the functioning of the system in which they are embedded. The pattern recognition system is implemented with Back propagation network and Hopfield network to remove the distortion from the input. The Hopfield network has high fault tolerance which supports this system to get the accurate output.
\end{abstract}

Keywords: Pattern Recognition; Hopfield network; Back Propagation Network; Training Set

\section{INTRODUCTION}

The Neural Network (NN) is nonlinear information processing systems that are designed from interconnected elementary processing devices known as neurons [1]. A NN could be a massively parallel distributed processor that includes a natural propensity for storing experimental data and creating it obtainable to be used. A Biological NN consists of a gaggle or teams of chemically connected or functionally associated neurons. One nerve cell may be connected to several alternative neurons and also the total range of neurons and connections during a network is also intensive. Connections known as synapses are typically fashioned from axons to dendrites. Natural nerve cells receive signals through synapses placed on the dendrites or membrane of the neuron. In most cases a $\mathrm{NN}$ is an adaptive system dynamical its structure throughout a learning part. $\mathrm{NN}$ are used for modeling advanced relationships between inputs and outputs or to seek out patterns in knowledge [2].

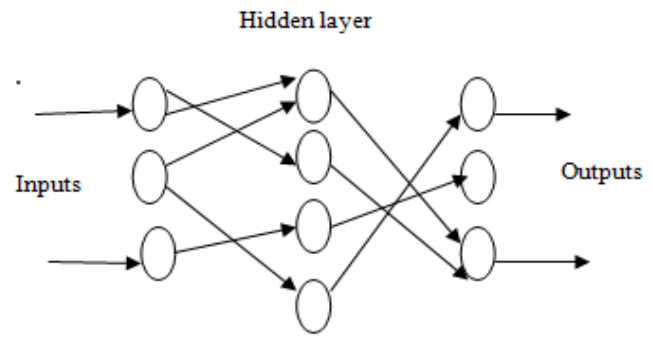

Figure 1 Basic network structure [3]

\subsection{Artificial neural networks}

The main characteristics of neural networks are that they need the power to find out advanced nonlinear inputoutput relationships, use successive coaching procedures, and adapt themselves to the information [2]. As ANN mature into industrial systems they're probably to be enforced in hardware. Their fault tolerance and reliability are so very important to the functioning of the system during which they're embedded [3]. This new approach to computing conjointly provides an additional graceful degradation throughout system overload than its additional ancient counterparts. ANN is an informatics system. During this information system the elements known as neurons process the data [1]. Artificial nerve cell is characterised by

- Architecture (connection between neurons)

- Training or learning (determining weights on the connections)

- Activation function

\subsection{Pattern recognition}

Machine recognition, description, classification, and grouping of patterns are necessary issues during a form of engineering and scientific disciplines like biology, psychology, medicine, marketing, pc vision, artificial intelligence, and remote sensing [2]. A pattern may be a fingerprint image, a written cursive word, a personality's face, or a speech signal. This recognition concept is straightforward and acquainted to everyone within the real world surroundings however in the world of AI, recognizing such objects is a tremendous accomplishment. The practicality of the human brain is wonderful, it's not comparable any artificial machines or software system. [4] The term pattern recognition encompasses a large vary of data process issues of nice sensible significance, from speech recognition and therefore the classification of written characters, to fault detection in machinery and diagnosis. The act of recognition will be divided into 2 broad categories: recognizing concrete things and recognizing abstract things [5]. The look of a pattern recognition system basically involves the subsequent 3 aspects: 1) knowledge acquisition and pre-processing, 2) knowledge illustration, and 3) higher cognitive process. The matter domain dictates the selection of sensor(s), preprocessing technique, illustration theme, and therefore the higher cognitive process model. It is usually in agreement that a well-defined and sufficiently unnatural recognition drawback can cause a compact pattern illustration and a straightforward higher cognitive process strategy.

\section{LITERATURE REVIEW}

Husam Ahmed Al Hamad [1] investigated and compared the results of 4 completely different artificial neural network models. Identical algorithmic rule was applied for all with applying 2 major techniques, 1st neural-segmentation technique; second apply a replacement fusion equation. The 
neural techniques calculate the arrogance values for every Prospective Segmentation Points (PSP) using the planned classifiers so as to acknowledge the higher model that increased the general recognition results of the written scripts. The fusion equation appraises every PSP by getting a fused value from 3 neural confidence values. CPU times and accuracies were conjointly reported. Jayanta Kumar Basu et. al., [2] proposed that among the various traditional approaches of pattern recognition the statistical approach has been most intensively studied and used in practice. The design of a recognition system require careful attention to the following issues: definition of pattern classes, sensing environment, pattern representation, feature extraction and selection, cluster analysis, classifier design and learning, selection of training and test samples and performance evaluation. New and emerging applications, such as data mining, web searching, retrieval of multimedia data, face recognition and cursive handwriting recognition, require robust and efficient pattern recognition techniques. The objective of this review paper was to summarize and compare some of the well-known methods used in various stages of a pattern recognition system using ANN. Fajri Kurniawan et. al.,,[3]presented a robust algorithm to identify the letter boundaries in images of unconstrained handwritten word. The proposed algorithm was based on vertical contour analysis. The Proposed algorithm was performed to generate presegmentation by analysing the vertical contours from right to left. The results showed that the proposed algorithm was capable to locate the letter boundaries accurately for unconstrained handwritten. Lupus Dung et. al., [4] planned that within the supervised coaching the author notice a collection of weights and biases for a pattern recognition neural network so as to classify all patterns in a very coaching knowledge set. But it might be tough if the neural network was not large enough for learning an oversized coaching knowledge set. During this paper the author planned a coaching technique and a style of pattern recognition neural network that wasn't massive however still able to classify all the coaching patterns precisely. The coaching technique facilitate the neural network to search out not only one but several sets of weights and biases for classifying all the coaching patterns, dominant the recognizing rejection and reducing the error rate. Dilruba et. $a l .$, , [5] urged that $\mathrm{NN}$ is as an efficient tool for pattern recognition. The success rate for recognizing known and unknown pattern was comparatively terribly high with compare to alternative techniques. This paper gift a comparative study of however $\mathrm{NN}$ classifies the patterns from coaching knowledge and acknowledges if testing knowledge holds that patterns. For learning from the coaching knowledge many approaches were gift among that the author had selected the back-propagation technique. Backpropagation rule in a very feed-forward network was used for the feature extraction. The author have used 2 approaches and network was trained with such that knowledge. The author supposed to search out the match quantitative relation of coaching Pattern to testing Pattern and therefore the result knowledge set found from the experiment also given within the paper. Zaheer Ahmad et. al., [6] planned that Urdu compound Character Recognition need sturdy techniques to develop as Urdu being a family of Arabic script was cursive right to left in nature and characters modification their shapes and sizes once they were placed at initial, middle or at the tip of a word. The developed system consists of 2 main modules segmentation and classification. Within the segmentation section pixels strength is measured to discover words in a very sentence and joints of characters in a very compound/connected word for segmentation. The most purpose of the system was to check the rule developed for segmentation of compound characters. Kauleshwar Prasad et. al., [7] focused on recognition of English alphabet in an exceedingly given scanned text document with the assistance of Neural Networks. It had varied applications that embrace reading aid for blind, bank cheques and conversion of any hand papers into structural text type. The primary step was image acquisition that non inheritable scanned image followed by noise filtering, smoothing and normalisation of scanned image, rendering image appropriate for segmentation wherever image was rotten into sub pictures. The author use character extraction and edge detection algorithmic program for coaching the neural network to classify and acknowledge the written characters. Binu P. et. al.,[8] wear down the popularity of hand written Malayalam character using wave energy feature (WEF) and extreme learning machine. The wave energy (WE) may be a new and sturdy parameter and was derived using wave rework. It might cut back the influence totally different varieties of noise at different levels. We tend to might reflect the WE distribution of characters in many directions at completely different scales. We tend to totally differ decomposition levels that have different powers to discriminate the character pictures. These options represent patterns of written characters for classification. This algorithmic program learned abundant quicker than ancient in style learning algorithms for feed forward neural network words. Dawei Qi et. al., [9] proposed that the edge detection problem in this paper was formulated as an optimization process that sought the edge points to minimize an energy function. An initial edge was first estimated by the method of traditional edge algorithm. The gray value of image pixel was described as the neuron state of Hopfield neural network. The state updated till the energy function touch the minimum value. The final states of neurons were the result image of edge detection. The novel energy function ensured that the network converged and reached a near-optimal solution. Ming-ai Li et. al., [10] planned a way to beat the multiple native minimum drawback of traditional distinction Hopfield neural network. On conditions that the changed Hopfield neural network works in a very parallel mode and its interconnection weight matrix was negative, it's only 1 stable state, and therefore the stable state will build its energy perform reach to its solely minimum. On the premise of the relation between the stability of the changed distinction Hopfield network and its energy function's convergence, the changed Hopfield network was applied to resolve LQ dynamic optimization management issues for time-varying systems. It can be made by building the equivalence between the energy performs of the changed Hopfield network and therefore the performance Index of controlled system. As a result, finding LQ dynamic optimization management drawback was reminiscent of operational associated changed distinction Hopfield network from any initial state to the stable state that represents the specified best management vector. The simulation results agree well with theoretical analysis.

\section{PROPOSED MODEL}

In the last few years neural network is found as an effective tool for pattern recognition. The Success rate has been examined for recognition pattern as well as unknown ones. It comes out to be comparatively very high. Back-propagation algorithm in a feed-forward network is used for the feature extraction. The Hopfield model of neural network working as an associative memory is chosen for recognition purposes [6]. The back propagation network and Hopfield network are combined together to get the appropriate result as more 
accurate than either using back propagation network or Hopfield network. The input to this network will be numeric characters, alphabets and special characters which will be recognized using the combination of back propagation network and Hopfield network. The distorted input is fed to the new combined network to get the accurate output. The Hopfield network is present to remove the distortion from the input and to get the exact output as the fault tolerance of Hopfield network is high as compared to Back propagation network.
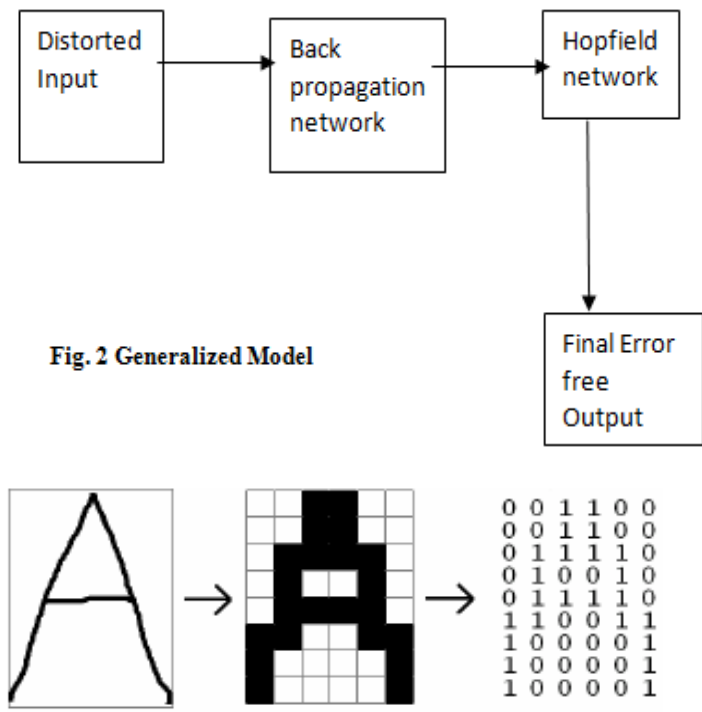

Fig 3 Input Characters

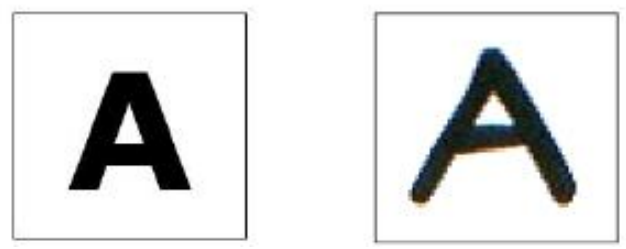

Fig 4 Output Character

\section{CONCLUSION}

In this present work we have implemented the Combined Network i.e. BPN and HP for pattern recognition of input patterns. In this work we have taken a sample of alphabet patterns to perform the Pattern recognition. As the initial step the image dataset is being maintained to represent different kind of character patterns. These images are trained using HP. The numbers of hidden layers are not fixed and are dependent on the complexity of the input. As fault tolerance of HP is more than BPN so the error calculating capability is more in HP. So the new defined network of HP and BPN is most suitable for recognizing the input pattern as compared to BPN. With distortion the accuracy level of output is more in new defined network as compared to only BPN. The output we get is similar to the trained dataset.

\section{REFERENCES}

[1] Husam Ahmed Al Hamad "Use an Efficient Neural Network to Improve the Arabic Handwriting Recognition"
International Conference on Systems, Control, Signal Processing and Informatics, Page no 269-274, 2013

[2] Jayanta Kumar Basu, Debnath Bhattacharyya and Taihoon Kim "Use of Artificial Neural Network in Pattern Recognition" International Journal of Software Engineering and Its Applications Vol. 4, No. 2, April 2010

[3] Fajiri Kurniawan, Mohd. Shafry Mohd. Rahim, Nimatus Sholihiah, Akmal Rakhmadi and Dzulkifli Mohamad "Characters Segmentation of Cursive Handwritten Words based on Contour Analysis and Neural Network Validation" ITB J. ICT, Vol. 5, No. 1, 2011

[4] Le Dung and Mizukawa M. "A Pattern Recognition Neural Network Using Many Sets of Weights and Biases", Computational Intelligence in Robotics and Automation, Page no 285-290,2007..

[5] Dilruiba, R.A., Chowdhury, N.Liza, F.F. and Kiarmakar "Data Pattern Recognition using Neural Network with BackPropagation Training “, Electrical and Computer Engineering, ICECE, Page no 451-455, 2006

[6] Zaheer Ahmad, Jehanzeb Khan Oraikzai and Inam Shamsher, "Urdu compound Character Recognition using feed forward neural networks,", International Conference on Computer Science and Information Technology, IEEE, pp.457-462, 2009.

[7] Kauleshwar Prasad, Devvrat C. Nigam, Ashmika Lakhotiya and Dheeren Umre "Character Recognition using Matlab's Network Toolbox" International journal service, Science and Technology Vol. 6, No. 1, page 13 February 2013

[8] Binu P, Chacko, Vimal Krishnan and G. Raju "Handwritten character recognition using wavelet energy and extreme learning machine" springer, International Journal of Machine Learning and Cybernetics, Volume 3, Issue 2, Page no. 149-161, June 2012

[9] Dawei Qi, Peng Zhang, Xuejing Jin and Xuefei Zhang "Study on Wood Image Edge Detection Based on Hopfield Neural Network", Proceedings of the International Conference on Information and Automation, IEEE, Page no 1942-1946, 2010

[10] Mingai Li, Jun-fei Qiao and Xiao-gang Ruan "A Modified Difference Hopfield Neural Network and its application" IEEE, Vol 1, Page 199-203, 2005 